This document is the accepted manuscript version of the following article:

Alderliesten, R. C., Brunner, A. J., \& Pascoe, J. A. (2018). Cyclic fatigue fracture of composites: what has testing revealed about the physics of the processes so far? Engineering Fracture Mechanics, 203, 186-196.

https://doi .org/10.1016/j.engfracmech.2018.06.023

This manuscript version is made available under the CC-BY-NC-ND 4.0 1icense http://creativecommons.org/1icenses/by-nc-nd/4.0/

\title{
Cyclic fatigue fracture of composites: What has testing revealed about the physics of the processes so far?
}

\author{
R.C. Alderliesten ${ }^{1}$, A.J. Brunner', J.A. Pascoe ${ }^{1,3}$ \\ ${ }^{1}$ Aerospace Structures and Materials Department, Faculty of Aerospace Engineering, TU Delft, The \\ Netherlands \\ ${ }^{2}$ Empa, Swiss Federal Laboratories for Materials Science and Technology, Dübendorf, Switzerland \\ ${ }^{3}$ Department of Astronautics, Imperial College London, South Kensington Campus, SW7 2AZ London, \\ United Kingdom
}

\section{Copyright notice}

This is the Accepted Author Manuscript version of this article. The version of record was published in Engineering Fracture Mechancis, and can be found via the DOI:

https://doi.org/10.1016/j.engfracmech.2018.06.023

In accordance with the Elsevier copyright policy, this version is released with the following copyright limitations: (c) 2018. This manuscript version is made available under the CC-BY-NC-ND 4.0 license http://creativecommons.org/licenses/by-nc-nd/4.0/

\section{Abstract}

Where for metallic materials fatigue fracture testing has contributed significantly to understanding macroscopic and microscopic fatigue failure, the understanding of fatigue fracture in fibre reinforced polymers (FRP) still seems limited. It appears that the research on fatigue in FRPs raises more questions rather than providing a framework for understanding the underlying mechanisms. Open questions include for example the correct formulation of the driving force and fatigue damage resistance, how to account for mixed mode loading and fibre bridging? But also the question to what extent averaging and homogenising over time- and length scales hinders the understanding in relation to the microscopic mechanisms?

To contribute to the development of understanding, this paper discusses several of these open questions. The selected questions were discussed at a recent workshop on 'Physics of fatigue damage growth' at TU Delft with participates from several laboratories covering expertise on fatigue fracture testing of metals and polymer composites. The aim of the discussion focuses on potential experimental and simulation approaches that may lead to a better understanding of the physics of the fatigue fracture process.

\section{Introduction}

Fatigue fracture testing has contributed significantly to understanding macroscopic fatigue fracture failure in metallic materials over the last 50 years, mainly due to work by Paris and co-workers [1-3]. Compared to this extensive work, which allows for successfully comparing different metallic materials with respect to their fatigue fracture behaviour based on empirical data presentations, the 
situation for understanding fatigue fracture in fibre-reinforced polymer (FRP) composites is quite different. In spite of extensive research, often based on tests originally developed for metals and adapted to FRP, there is only one standard fatigue fracture test procedures available, i.e., ASTM D6115 [4]. Most research recently presented in literature has adopted quasi-static standardized tests and specimens (e.g. ASTM D5528 [5] or ISO 15024 [6] for mode I, ASTM D7905 [7] or ISO 15114 [8] for mode II, ASTM D6671 [9] or a modified C-ELS procedure (based on ISO 15114) but with the specimen inverted for a variable and fixed-ratio mixed-mode I/II test, respectively, but all with cyclic instead of quasi-static loading applied.

However, the differences in adopted test methods and in particular the analysis procedures, seem to result in more questions rather than providing a framework for understanding of the underlying mechanisms. For example, what are the assumptions underlying the cyclic fatigue fracture (mechanics) analysis procedures? The measurements for analysis comprise load, displacement and delamination length, analogous to quasi-static tests, while ignoring stochastic occurrences of microscopic, localized cracks on the ply-level scale or below, which may be affected by local stress fields (including internal residual stresses) differing from the stress intensity assumed from the applied global loads. Hence, the lack of such a framework of understanding subsequently hinders the application of test data in materials development and structural design.

Take for example the ongoing discussion on the correct formulation of the driving force for, and resistance to, fatigue crack growth, e.g. [10-13]. To apply data in engineering predictions the principle of similitude has been adopted, but for successful application of this principle, agreement is required on which parameters uniquely describe similitude. Both the stress intensity factor (SIF) and the strain energy release rate (SERR), originating from linear elastic fracture mechanics, have been used. However, there is no consensus on the formulation, i.e., whether it is more appropriate to use $\mathrm{G}_{\max }, \Delta \mathrm{G}$, or $\Delta \sqrt{ } \mathrm{G}$ or combinations thereof. Without the proper basis for evaluating data, other questions then also lack a satisfying answer, like questions on how to take loading mode-mix into account [14], or on the assessment of fibre bridging [15].

In addition, when reviewing literature on fatigue fracture in metallic materials and in FRPs, it appears that occasionally similar trends are reported. It is therefore worthwhile to ask where similarities between fatigue fracture behaviour and underlying physics of metals and FRP composites exist or whether the empirically observed analogies are accidental.

Furthermore, it seems that the microscopic mechanisms in both material classes are not sufficiently understood yet. The empirical approaches for data analysis and interpretation used so far (as the double-logarithmic Paris-type graphs) consider material behaviour averaged and hence homogenized over time- and geometrical scales. For large portions of a typical fatigue test, the expected crack extension in a single cycle may be on the order of $10^{-4}$ to $10^{-6} \mathrm{~mm}$, which cannot be resolved by the methods traditionally used to measure crack length during a fatigue crack growth experiment. Instead, the crack growth rate is determined from crack lengths measured at intervals of a few hundred to several thousand cycles. A recent review re-analysing data from many publications on fatigue crack growth of metals [16] addresses the role of the materials' microstructure in cyclic fatigue growth and summarizes (cite) "The experimental data presented in this paper reveals that even if the growth of long cracks in two materials, with different microstructures, have different $\mathrm{da} / \mathrm{dN}$ versus $\Delta \mathrm{K}$ curves the corresponding small crack curves can be 
similar. We also see that long cracks in a large range of steels with different microstructures, chemical compositions, and yield stresses can have similar crack growth rates." While these empirical facts are supported by the data, there is currently no understanding of the underlying physical reasons for this behaviour. For FRP, the question of whether there are analogous effects due to "small" or "short" crack (see, e.g., [17] for definitions) remains open. This clearly illustrates that the microscopic processes in fatigue fracture are far from being understood.

The aim of the present paper, therefore, is to identify open questions and to discuss potential experimental and simulation approaches that may lead to a better understanding of the physics of fatigue fracture. In particular, better understanding the underlying physics of scatter will show which extrinsic sources of scatter must be eliminated by better experimental procedures, and will allow quantifying intrinsic sources of scatter. Clearly, quantitative assessment of scatter in the test data constitutes essential information for structural design. After all, designers must account for potential material variability in their designs, however, without applying too conservative limits. With respect to using fatigue delamination test data obtained from materials coupons to life prediction of composite structures, another recent paper [18] concluded (cite) "... experimental data suggest that the fastest growing, i.e. lead, delaminations that arise under cyclic fatigue loading of real structures or components from mis-drilled holes, ply drop-offs and impact damage show no, or only little, retardation. Such retardation typically arises due to fibre bridging developing across the faces of the delamination as the fatigue crack advances. Therefore, of course, the Fatigue Crack Growth data that is ascertained in the laboratory, and then employed as a material allowable property to design and life the structure, as well as for the characterisation and comparison of composite materials, must also exhibit no, or only minimal, retardation." The concept of the so-called "lead crack" has been further discussed by Molent and Forrester [19] and it was concluded (cite) "the growth of some (limited) composite laminate and bonded repair damage types was reviewed and found to broadly conform to the lead crack framework. Thus equations originally derived for metals may have utility for the assessment of the growth of defects in composite structures. One such equation is the Hartman-Schijve variant. With further research (and data) a damage-tolerance approach appears to be feasible and could be used to comply with the 2009 US Federal Aviation Administration (FAA) slow growth approach to certifying composite, adhesively bonded structures and bonded repairs."

That fatigue fracture data from materials coupon testing may allow for prediction of fatigue crack growth in operational composite structures or components such as fighter aircraft is important for the engineering design as well as inspection or monitoring and maintenance of composite structures in general. Nevertheless, the approaches discussed by $[18,19]$ are based on equations that have essentially been derived empirically and use data for which the sources of scatter have not yet been fully elucidated. A sufficient understanding of scatter in FRP materials data used for composite structural design or life-time prediction is important and a statistical description of scatter is required by composite design guidelines, see, e.g., [20, 21].

Improvements in physics based understanding of fatigue fracture in FRP laminates and the related scatter very likely require a deeper insight into the underlying microscopic mechanisms. Pointing that out and providing a base for further discussion of these issues is the goal of this paper. 


\section{Formulating similitude}

A variety of prediction models adopting fracture mechanics have been developed in the past decades. The literature illustrates that most models have been developed in a purely empirical fashion. A fracture mechanics parameter was taken to describe similarity and using this parameter, observed data was phenomenologically correlated and formulated as material characteristic, e.g., in the form of Paris-type relationships [1-3] or other forms, e.g., the Hartman-Schijve relation [22]. These empirical relations (eqn. 1) generally have the form of a Paris-type power-law

$$
\frac{\mathrm{da}}{\mathrm{dN}}=\mathrm{C} \Delta \mathrm{K}^{\mathrm{n}} \quad \text { or } \quad \frac{\mathrm{da}}{\mathrm{dN}}=\mathrm{C} \Delta \mathrm{G}^{\mathrm{n}} \quad \text { or } \quad \frac{\mathrm{da}}{\mathrm{dN}}=\mathrm{CG}_{\max }^{\mathrm{n}} \quad \text { or } \quad \frac{\mathrm{da}}{\mathrm{dN}}=\mathrm{C} \Delta \sqrt{\mathrm{G}}^{\mathrm{n}}
$$

where $\mathrm{da} / \mathrm{dN}$ is the crack growth rate [mm/cycle], $\Delta \mathrm{K}$ is the stress intensity factor (SIF) range $[\mathrm{MPa} \vee \mathrm{m}]$ and $\mathrm{G}$ the strain energy release rate (SERR) $\left[\mathrm{N} / \mathrm{mm}\right.$ or $\left.\mathrm{mJ} / \mathrm{mm}^{2}\right]$, and $\mathrm{C}$ and $\mathrm{n}$ are curve fitting parameters, which each must have a physical meaning. Dimensional analysis highlights that the constants $C$ have units that are a function of the exponents' value $n$, which obscures the physical meaning of $\mathrm{C}$. This physical meaning, in fact, has never received a proper explanation.

In discussing these models, Rans et al. [11] illustrated that when taking $G_{\max }$ or $\Delta G=\left(G_{\max }-G_{\min }\right)$ as similitude parameter, the stress ratio effect in the crack growth resistance curves is opposite to what is commonly observed for metals when plotting the crack growth rate against $\Delta \mathrm{K}=\left(\mathrm{K}_{\max }-\mathrm{K}_{\min }\right)$. Using $\Delta \sqrt{ } G=\left(\sqrt{ } G_{\max }-\sqrt{ } G_{\min }\right)$ results in a similar stress ratio trend as observed for metals, see Figure 1, because of the correlation between $\mathrm{G}$ and $\mathrm{K}^{2}$.

To account for the effect of stress ratio in mode I fatigue loading, two-parameter models have been proposed in which both the cyclic SERR and the maximum SERR describe similitude together [23-25]. These models commonly result in a single crack growth resistance curve, collapsing all data for different stress ratios. Although most models multiply the two SERR components each with their own exponent, Khan et al. [26-28] argued based on fractographic evidence that it might be more appropriate to superimpose both components. An alternative formulation adopting both SERR components is represented by the Hartman-Schijve equation [18, 22] allowing to collapse most data to a single curve as well. However, none of these models ever received a proper explanation for the physical basis underlying the formulation. In addition, all models are characterised by the values for constants and exponents being selected purely on the apparent fit to a single curve.

It can be added that single curves described by, e.g., exponent and slope as in the Paris relation, or described by additional parameters, as, e.g., in the Hartman-Schijve relation, still remain empirical and limited in their representation of the material behaviour under fatigue fracture. Here, one could argue that such empirical approaches are sufficient for practical engineering purposes, but will not provide physical understanding of relevant effects. However, even for comparative material characterization, e.g., for selecting the optimal laminate for the application, the position of the average fitting line provides only one part. The second part is the scatter associated with the data, and this is of specific importance if the data are used in structural design. When comparing materials, a Carbon Fibre Reinforced Polymer (CFRP) composite with "less" scatter in the crack growth resistance curve (yielding a lower bound when scatter is considered in the analysis) than another CFRP composite with a lower crack growth resistance curve, but with more scatter, may be the preferred option. 
Hence, questions remain whether the macroscopic basis for fatigue fracture in composites is fully understood, and whether observed trends have a physical basis or merely, possibly to a certain extent only, represent artefacts of the empirical model or of the test and analysis procedure selected. One should consider that despite fracture mechanics being based on physics, using fracture mechanics parameters such as, e.g., $\Delta \mathrm{K}=\left(\mathrm{K}_{\max }-\mathrm{K}_{\min }\right)$ and relating these empirically or phenomenologically to crack growth observed in experiments does not necessarily imply that the physics is satisfied in the analysis.

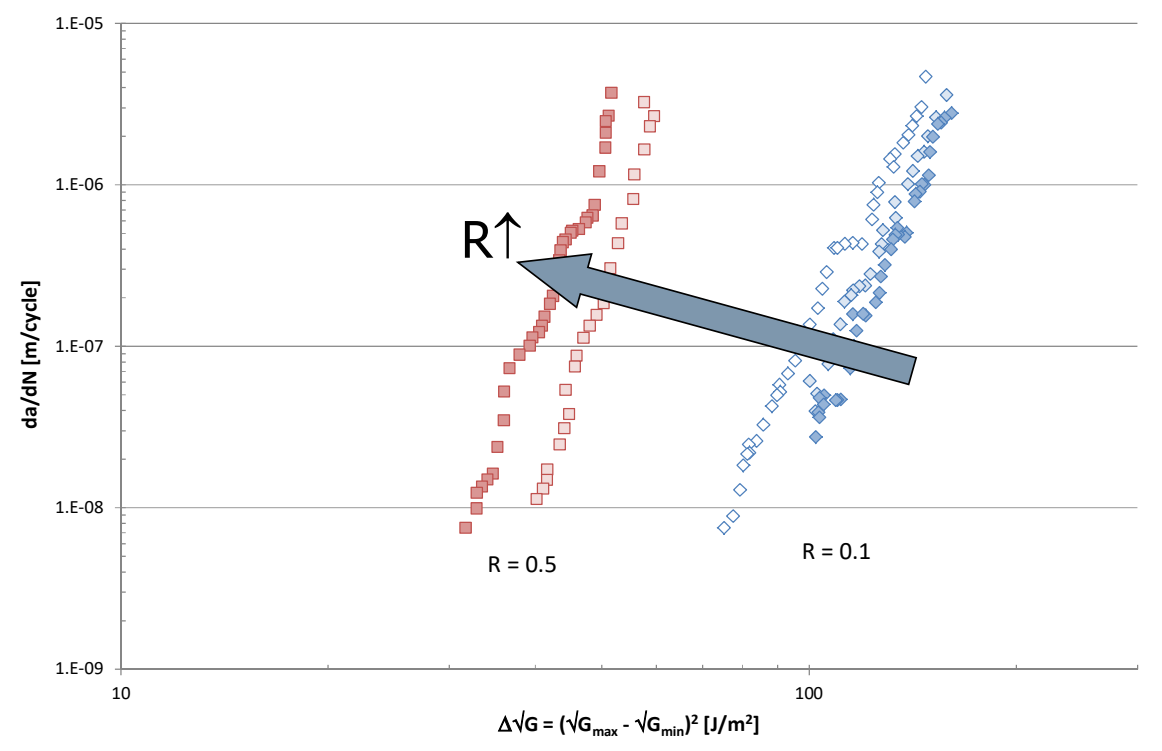

Figure 1 Stress ratio effect in unidirectional laminates at the $0^{\circ} / 0^{\circ}$ interface; increase in stress ratio shifts the curves as indicated when $\Delta \sqrt{ } \mathrm{G}$ is taken as similitude parameter [newREF29]

\section{Sources of scatter}

This discussion of the proper similitude becomes important when minimizing the amount of scatter observed in the experimentally obtained fatigue fracture data. In the assessment of scatter in crack growth data, one should distinguish two categories of scatter, i.e. intrinsic and extrinsic scatter. Examples of the latter are test set-up (e.g., compliance or play in the load-introduction, or load cell range with insufficient measurement resolution,), operator experience (e.g., learning curve for proper test set-up as well as visual observation during testing [30,31]) , but also machining variation in specimen width and cutting quality, or variation in laminate plate thickness (e.g., near the edges) affecting the individual specimens' compliance [32] which should be limited or excluded by proper test specification.

The intrinsic scatter, i.e. due to inhomogeneous material morphology and process variability, should be considered relevant for design purposes and shall not be excluded nor underestimated. FRP composite processing can result in defects such as voids or porosity, inclusion of foreign particles, or local fibre-matrix debonds, but can also affect fibre alignment and produce residual stresses, especially with improper cure (e.g., [33-34]) that may all interfere with the applied global stresses and the resulting delamination propagation. The influence of fibre bridging on the delamination 
growth characteristics measured in unidirectional fibre reinforced double cantilever beam specimens $[5,6]$ in the view of the authors constitutes a source of intrinsic rather than extrinsic scatter, since it results from the specific unidirectional fibre lay-up chosen for the test specimens. The difficulty in using data from standardized procedures usually specified for unidirectionally fibre-reinforced laminates for structural design is then accounting for the different behaviour of the actual laminate lay-up used in the design, not to mention additional complications such as stress concentrations produced by the geometry of actual parts. Avoiding unidirectional lay-up in the FRP fatigue fracture test specimens, at least around the path of delamination propagation, may prevent or at least reduce fibre bridging compared with the unidirectional lay-up of the same FRP composite, but can yield different fracture morphology or even multiple cracks or crack branching as shown by studies on multi-directional or angle-ply laminates, e.g., [35-38]. Therefore, the transferability of test coupon data to structures either requires accounting for differences in the amount of fibre-bridging between the test coupons and the lay-up used in the structures or coupon testing with structure-specific layups. The latter, however, may not satisfy the symmetry, balance and bending-stiffness requirements for DCB specimens, e.g., noted in $[5,6]$.

However, the view on the fibre bridging being an extrinsic source of scatter may not be beyond dispute due to complex questions related to fibre bridging. Take for example the experimental results presented by Yao et al. [15], who have illustrated that fibre bridging has a substantial influence on the position of the obtained crack growth resistance curve, see Figure 2 . What can be learned from this observation is that apparently, a variation in the initial pre-crack length of \pm 1 or \pm 2 $\mathrm{mm}$, easily results in an amount of scatter of the order currently observed in most data reported, which could be considered extrinsic scatter. This trend is also shown by data from G-constant tests presented by Hojo and Aoki [39]. Standardization of testing requires therefore a thorough assessment of all parameters with respect to scatter, not necessarily limited to accuracy in load and displacement measurements only.

With the current trend in using effective crack length approaches (as e.g.,for quasi-static mode II [8]) derived from compliance rather than visual observation of delamination length, the scatter in load and displacement measurements will affect both axes of the double-logarithmic plots in the Parisand Hartman-Schijve-type analysis. Although this does not necessarily induce scatter in the results, basing compliance on peak values only will impose scatter: it will include effects of measuring equipment not being correctly zeroed, and of any play in the set-up, assuming the forcedisplacement curve to pass through the origin. Although play in set-up might be accounted for as extrinsic scatter with compliance calibration with a sufficiently stiff dummy specimen, the difference between compliance slopes through zero and maximum load, and through minimum and maximum load will affect the data analysis. Similarly, the assumption of linear compliance calibration curves where it effectively is non-linear will introduce (intrinsic) scatter. 


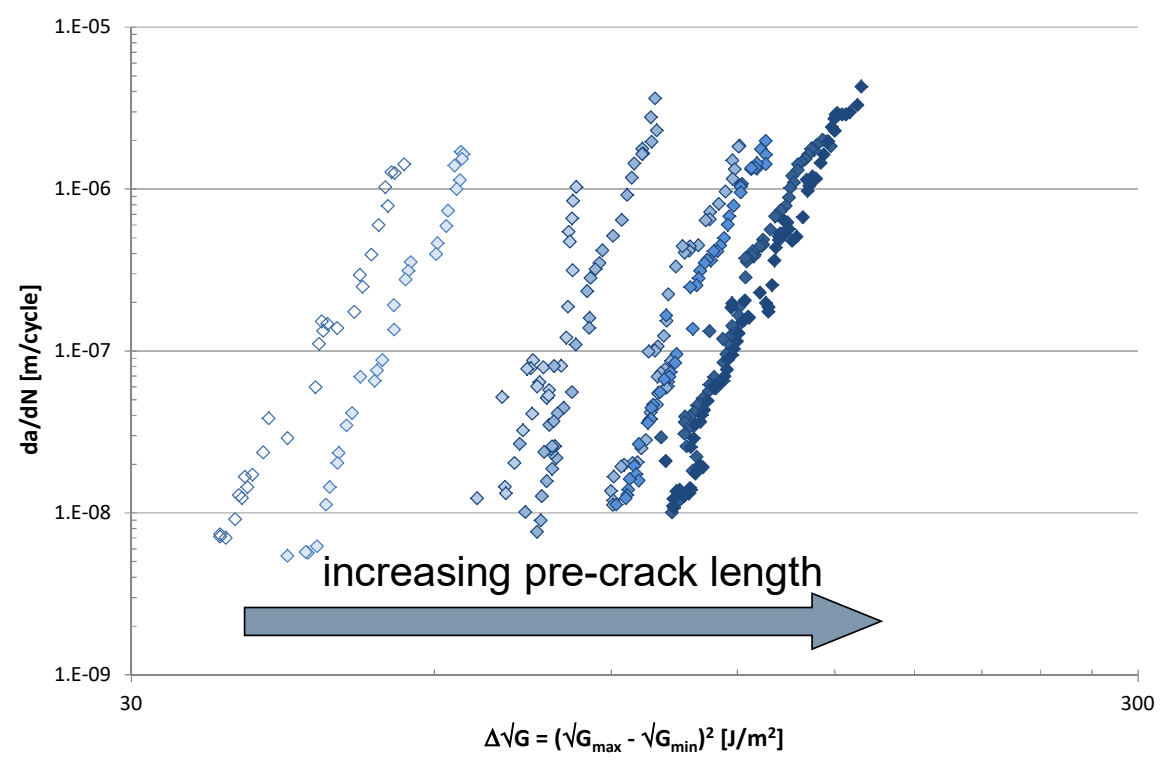

Figure 2 Shift in crack growth resistance curves with increasing pre-crack length illustrating the effect of fibre bridging [15]

\section{Fracture mechanics versus physics}

Looking at the fatigue fracture process from a physics perspective implies reconsideration of the energy balance that Griffith originally proposed [40]. Fatigue loading constitutes the application of cyclic work of which a certain amount is dissipated through various fracture processes. The macroscopic physics can be quantified, by measuring both forces and displacements throughout the load cycle, and plotting the resulting $\mathrm{dU} / \mathrm{dN}$ against the measured crack growth rate $\mathrm{dA} / \mathrm{dN}[12,15$, 41]. The crack growth rate $d A / d N$ refers to a crack surface area $A$ rather than a crack length a, because physically fracture surfaces are created. The slope through any point of the obtained curve and the origin represents the effective resistance to crack growth described by $G=d U / d A$. Hence, the governing physical equation is given by

$\frac{\mathrm{dU}}{\mathrm{dN}}=\frac{\mathrm{dU}}{\mathrm{dA}} \frac{\mathrm{dA}}{\mathrm{dN}}$

This approach immediately reveals a characteristic difference between both evaluation approaches; where in fracture mechanics the SERR $G=d U / d A$ constitutes a crack driving force, i.e., a measure of strain energy release if a crack extends, in physics it represents a consequence, i.e., the energy release as result of crack extension. This latter concept constitutes the material's resistance to crack growth.

As a result, common graphical presentations plotting da/dN against SERR parameters imply plotting a measurement against a model, whereas graphs following the concept of equation (2) imply plotting measurement against measurement. The latter, therefore, should be considered more appropriate when physically characterizing fatigue fracture in composites. 
Because equation (2) does not contain any parameter quantifying the applied work or strain energy, capturing the material's resistance, expressed as $\mathrm{dU} / \mathrm{dA}$, separate from the applied work, implies one distinguishes between driving force and resistance explicitly.

One advantage of separating the driving force (or work) from the physical resistance is that it allows for better correlation with microscopic observations on the fracture surfaces. For example, Amaral et al. [42] illustrated how the increasing resistance measured in a carbon / epoxy composite M30SC150-DT 120-34 F correlates well with the fractographic observations, see Figure 4.

In addition, the distinction between driving force and resistance has revealed that fibre bridging constitutes not only a shielding mechanism, i.e., reducing the driving force available at the crack tip by carrying part of the applied load, but also contributes to resistance: strain energy is dissipated when bridging fibres are pulled out of the matrix or when they fail. This is illustrated by Yao et al [43] where data plotted following equation (2) also shifts to a certain extent until full saturation of bridging is achieved.
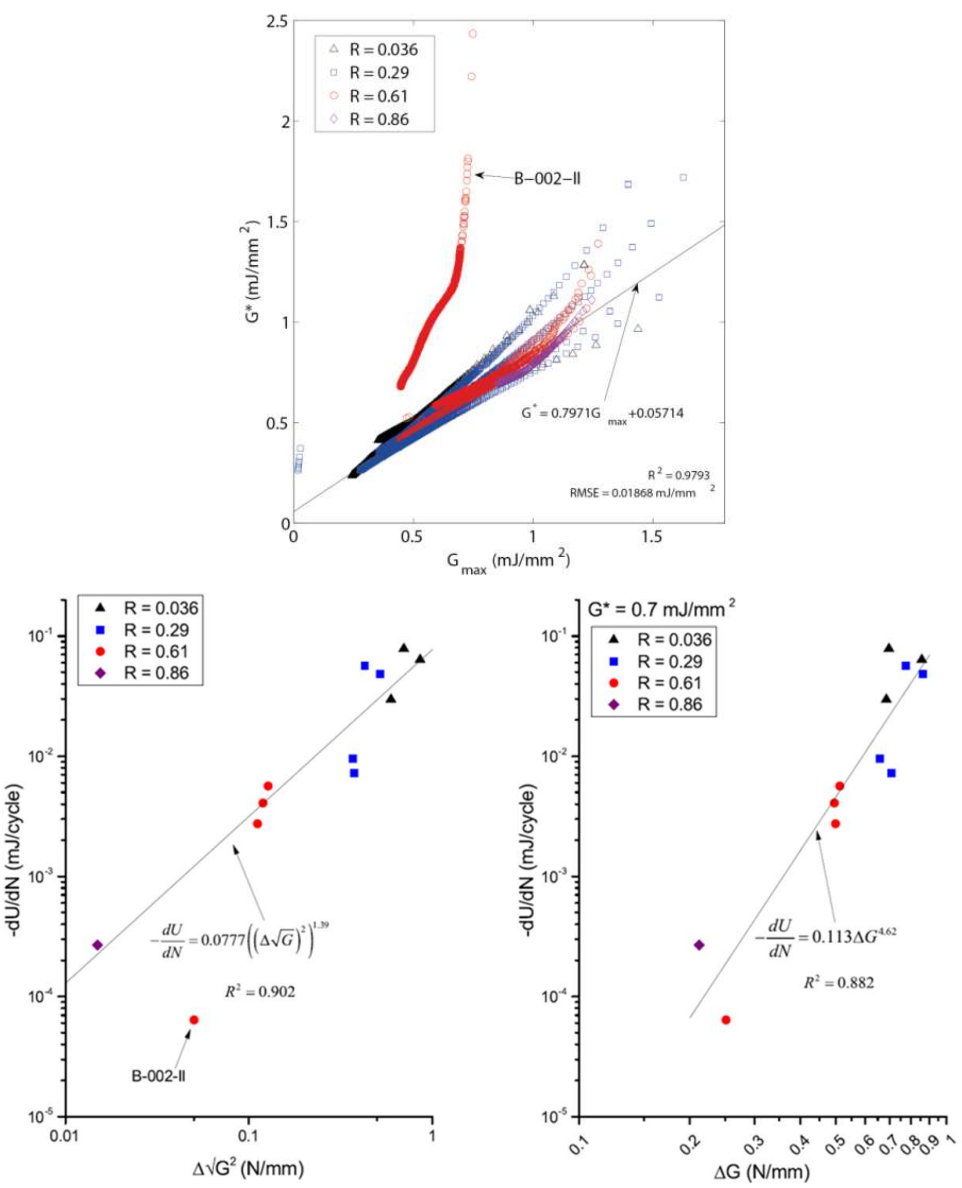

Figure 3 Correlation between the effective resistance $\mathrm{G}^{*}$ and $\mathrm{G}_{\max }$ (left) and the crack driving force with $\Delta \sqrt{ } \mathrm{G}^{2}$ (centre) and with $\Delta \mathrm{G}$ (right) [newREF41]

\section{Correlating physics with fracture mechanics}


With the concept of available energy for crack growth (driving force) and the effective resistance against fracture $(\mathrm{dU} / \mathrm{dA})$, fracture mechanics models can be evaluated for their physical relevance. In that respect, Pascoe $[41,44]$ has illustrated how the effective resistance correlates well with the maximum SERR $\left(G_{\max }\right)$ used in fracture mechanics, while the strain energy dissipation correlates best with $\Delta \mathrm{G}$, see Figure 3 . With that correlation in mind, it can be reasoned that for example the Hartman-Schijve equation given by [22]

$$
\frac{d a}{d N}=D\left[\frac{\Delta \sqrt{G}-\Delta \sqrt{G_{t h r}}}{\sqrt{1-\frac{\sqrt{G_{\max }}}{\sqrt{A}}}}\right]^{n}
$$

explicitly includes terms correlating to the driving force in the numerator, i.e. $\Delta \sqrt{ } G$, while the terms correlating to resistance are placed in the denominator, i.e. $\sqrt{ } \mathrm{G}_{\text {max }}$. The threshold term $\sqrt{ } \mathrm{G}_{\text {thr }}$ may then be considered a minimum driving force necessary in order to have crack growth.

An analogy would be the case of pushing a box across the ground. The net driving force on the box is equal to the pushing force, minus the sliding friction. The acceleration of the box is determined by the ratio of the net driving force to the box' resistance to movement, i.e its inertia, which is represented by the physical quantity of mass. So in the case of the Hartman-Schijve equation $\Delta \sqrt{ } \mathrm{G}$ $\Delta \sqrt{ } G_{\text {th }}$ can be thought of as representing the net crack driving force, while $\sqrt{ } G_{\max }$ is a measure of the material's resistance to crack growth. One should note here that the use of $\sqrt{ } \mathrm{G}$ rather than $\mathrm{G}$ in equation (3) corresponds to original, but empirical formulations expressed in $\mathrm{K}$ [45] following the original Paris equation [1-3].

The question remaining then, however, is how to interpret the physics with equation (3) when $D$ and $\mathrm{n}$ are 'arbitrarily' selected to fit the data?

Unless similitude is taken in agreement with physics, one could still question parameters like the threshold SERR for their physical meaning: does $\mathrm{G}_{\text {thr }}$ represent a physical mechanism, or is it merely an artefact of representation? Indifferent of whether one prefers to execute the DCB tests in load or displacement controlled conditions: could $\mathrm{G}_{\mathrm{thr}}$ be an artefact of load cell accuracy? Until now, the value of $G_{\text {thr }}$ is selected purely on the apparent fit of data to a single curve, assumed to be approximately straight in a double-logarithmic graph, i.e., fitting the data to a power-law type of equation. In other words, $G_{\text {thr }}$ is treated as a fitting parameter, whereas if it is related to a physical mechanism, it should be possible to determine $G_{\text {thr }}$ independently.

\section{Physical basis for corrections and scatter}

In fracture mechanics concepts similitude parameters are often related to the stresses imposed by the mechanical fatigue load cycle. The recorded crack growth rate $\mathrm{da} / \mathrm{dN}$ is then plotted against the SERR, which is solely based on calculations using relations from compliance calibration or modified beam theory. External influences like small fluctuations in ambient temperature by \pm 1 or $\pm 2^{\circ} \mathrm{C}$ are neglected and not incorporated in quantifying the driving force. 
Physically, these external aspects can be accounted for when quantifying the amount of work applied. Take for example the standard fatigue crack growth tests for metallic centre crack tension specimens. The SIF is corrected with a finite width correction factor, which mostly has been derived from theoretical stress analyses.

When quantifying the applied work through measuring the applied forces and displacements however, one can illustrate that none of these theoretical corrections are correct if that fatigue test has been executed in an environment where the ambient temperature varies by a few degrees. Similar effects can be observed when performing fatigue fracture testing in composites. Either the effects are ignored, putting a requirement on the test to avoid any temperature fluctuation larger than about $1^{\circ} \mathrm{C}$, or one quantifies the applied work throughout the entire test to filter out this effect that appears otherwise in the graph as scatter.

\section{Quantifying physical fatigue damage}

In establishing a proper physical basis for characterisation of fatigue fracture, one has to carefully review whether the concept of 'crack length' as one dimensional, correctly captures the physical damage created in fatigue fracture $[28,46]$. To a certain extent this was discussed by Amaral et al [42] when correlating fractographic observations to the physical resistance measured on a carbon/epoxy composite (M30SC-150-DT 120-34 F). An increase in resistance seems to correlate to an increase in damage features on the fracture surface, see Figure 4.

However, visual features on the fracture surface at best represent a qualitative measure of damage. Quantifying the energy dissipation for individual features is required to fully develop the physical equation. 


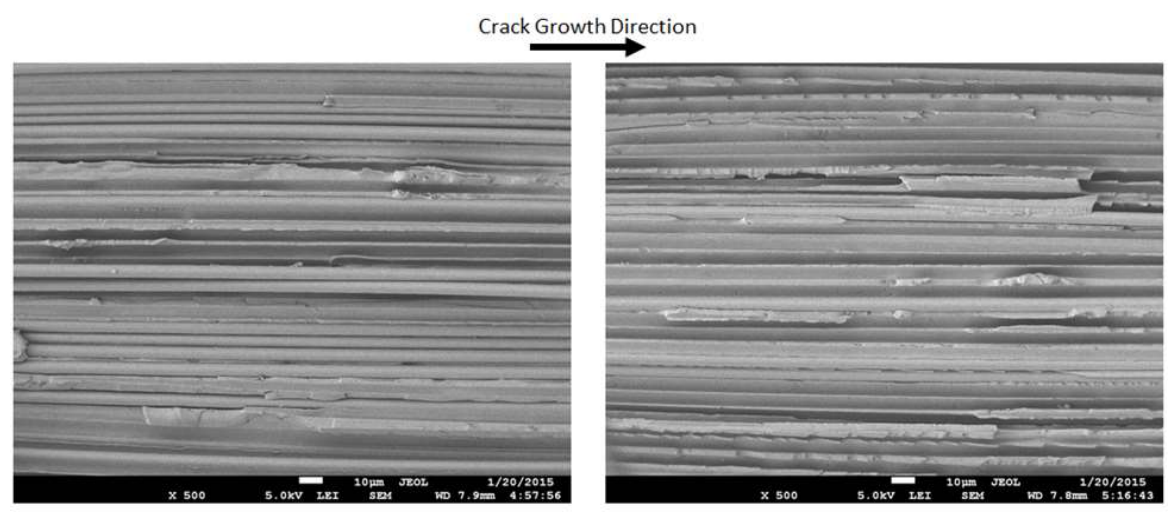

(a) Fatigue fracture surface $d a / d N=0.05 * 10^{-6} \mathrm{~m} /$ cycle

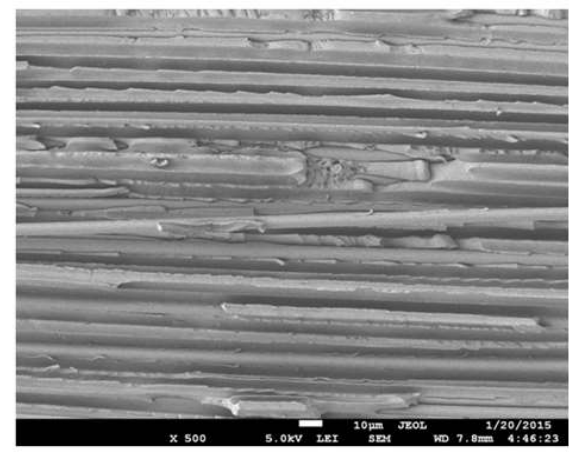

(b) Fatigue fracture surface $d a / d N=0.1 * 10^{-6} \mathrm{~m} /$ cycle

(c) Fatigue fracture surface $d a / d N=0.5 * 10^{-6} \mathrm{~m} /$ cycle

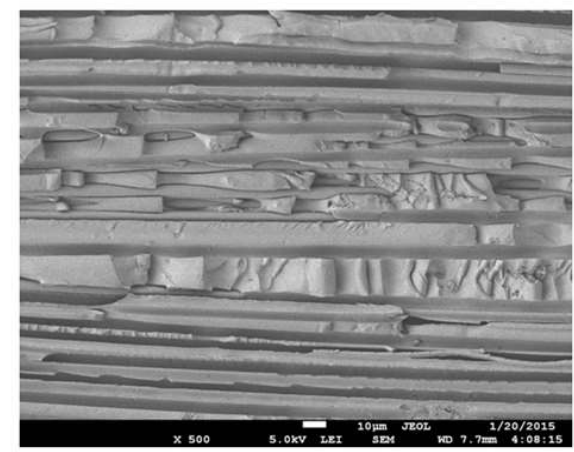

(d) Fatigue fracture surface $d a / d N=2 * 10^{-6} \mathrm{~m} /$ cycle

Figure 4 Increase in damage features on the fracture surface correlating to an increase in measured effective resistance dU/dA [newREF42]. Note that the applied load cycle (in terms of $G_{\max }$ and $\Delta G$ ) was different for each picture, see for example Figure 1 and 2.

Here, recent developments in non-destructive testing techniques may aid in understanding the microscopic processes in relation to energy dissipation. Take for example the application of the Acoustic Emission (AE) technique. Pascoe [41] reports AE measurements during fatigue loading of adhesively bonded DCB specimens, illustrating that the number and intensity of received acoustic signals differs over the individual load cycles, see Figure 5. Such measurements may shed light on the appropriateness of averaging experimental results over time and length scales. The crack growth rate $\mathrm{da} / \mathrm{dN}$ is often obtained by averaging over a sequence of constant amplitude load cycles, while discretizing AE measurements to individual load cycles reveals very discontinuous crack growth.

Note that most AE signals illustrated in Figure 5 near the minimum of the load cycle are considered here of less relevance, as they may relate to other sources like friction noise related to crack closure rather than to fracture mechanisms. This distinction was performed based on waveform mostly, as reported by Pascoe [41] and in more detail by Amaral et al [47]. The exact source of these low amplitude signals at low stress levels remains to be further studied. The relevant $A E$ signals seem to occur stochastically at various levels in the load cycle, i.e. fracture does not always happen at similar levels in the load level. 


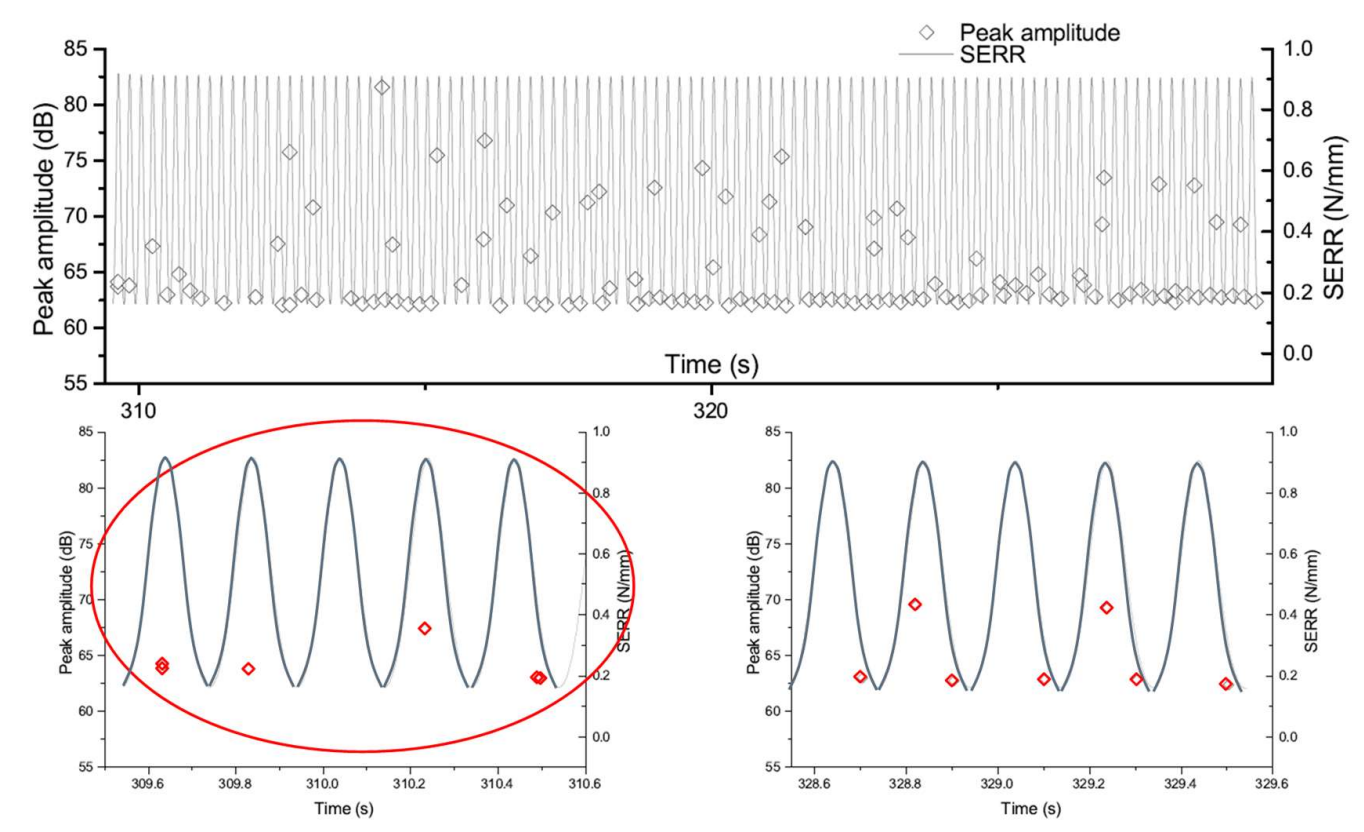

Figure 5 Illustration of measured hits during fatigue loading using the AE technique; the number and intensity of hits differs over the individual load cycles. The upper panel shows the full range of data, the lower panels show the first and last five cycles respectively. [newREF41]

$\mathrm{X}$-ray projection radiography using a contrast agent to highlight the delamination, combined with insitu AE monitoring of quasi-static mode I fracture in a recent analysis [48] yielded rough estimates of the size (diameter) of the individual micro-cracks generated during the mode I loading of AS4/PEEK DCB-specimens. These micro-cracks are likely the sources for the AE signals recorded. Estimating the total fracture surface, i.e., the delaminated area, from radiography, an average crack size between about 50 up to about $200 \mu \mathrm{m}$ was obtained for the AE signals with amplitudes in the range between about 60 and $80 \mathrm{~dB}_{\mathrm{AE}}$. Further, the radiography images indicated the occurrence of these microcracks at stochastically distributed locations along the tip of the delamination. The sum of these many micro-cracks then constitutes the observed quasi-static delamination propagation. The visual observation of delamination length increase on the edges of the DCB-specimens according to standard fracture test procedures $[5,6]$ does not capture the microscopic and stochastic nature of the underlying physical damage mechanisms.

It can be hypothesized that the same type of microscopic damage mechanism is also causing the delamination propagation under cyclic fatigue fracture loading, even though reports on in-situ fatigue fracture induced crack length characterization by non-destructive test methods such as X-ray imaging are scarce. One example of fatigue induced cracks in double-notched CFRP-epoxy specimens of different toughness is discussed in $[49,50]$. Crack lengths seem to be on the order of a few millimeter (roughly 3-5 mm) after 1000 cycles and less than $10 \mathrm{~mm}$ after $100 \mathrm{kcycles}$. A quantification of the micro-crack size is difficult with these data, but crack sizes on the order of micrometer would seem to be consistent with that. 
The AE recorded and discussed by Pascoe $[41,51]$ noted above, also provides support for this hypothesis. Assuming a similar size for cyclic mode I fatigue induced micro-cracks as that estimated for quasi-static mode I fracture then leads to the conclusion that at very low delamination propagation rates $\mathrm{da} / \mathrm{dN}$ near threshold (i.e., on the order of $10^{-8} \mathrm{~mm} / \mathrm{cycle}$ ) likely require many cycles for just one micro-crack to form. Note that a length of $10^{-8} \mathrm{~mm}$ corresponds to $0.01 \mathrm{~nm}$, which is on the order of a single atomic radius. One may therefore wonder if the crack is truly advancing by a single atom every cycle. It seems more likely that these low crack growth rates should be interpreted as the averaged result of stochastic processes, which create larger crack growth in some cycles, and no crack growth in others.

This in turn leads to the question, how much the fatigue micro-crack formation may be affected by local stress fields, including internal / residual stresses from CFRP laminate manufacturing and processing compared to the nominally applied global stresses from the test machine. It could be plausible that internal stresses may contribute an additional source of scatter for the delamination length measurement on the microscopic scale. It could further be questioned whether the apparent stochastic occurrence of micro-cracks really holds or whether local stress fields may yield subtle cooperative effects.

In addition, the question of the typical average size (diameter) of micro-cracks created by cyclic fatigue fracture loading in CFRP, there is the question of the time-scale on which these cracks occur. For estimating realistic physical time-scales of the source phenomena, i.e., the cracks forming, recorded AE signals (waveforms) yield a so-called rise-time which can be considered to constitute a first upper bound on the source mechanism time-scale. The AE rise-time is the duration between the moment when the recorded signal first exceeds the acquisition threshold and the time when the maximum signal amplitude occurs. The AE signal rise-times measured in laboratory-scale CFRP specimens are roughly between a few tens and about one-hundred microseconds. However, these values are measured after wave propagation in the material, likely modulated by attenuation and dispersion effects, and also after conversion to an electric signal in the piezo-sensor with possible, additional filtering. To date, it seems that "rise-times" at the signal source, i.e., the time over which the source mechanism is active in the source volume have never been successfully measured [52]. For modelling AE signals caused by crack formation in a standard unidirectional CFRP specimen under flexural load [53] the source excitation time was varied between 50 ns and 3 microseconds [54]. The AE signals thus simulated by the COMSOL Multiphysics Structural Mechanics Module (see [55]) for matrix cracking and fibre breaks agreed reasonably well with experimental observations. This indicates that source rise-times on the order of one microsecond or less are realistic for microscopic damage mechanisms in CFRP. Therefore, formation of the individual micro-cracks presumably occurs on length and times scales far below those used in monitoring the cyclic fatigue fracture tests for determination of the average delamination rate da/dN per cycle. This stochastic micro-crack formation will also contribute to some scatter in the essentially macroscopic crack size and growth averaging for the data analysis.

In cyclic fatigue fracture tests of CFRP, it is recommended to use loading frequencies between about 1 and $10 \mathrm{~Hz}$. If higher frequencies are used, the specimen temperature shall be monitored and, if exceeding a specified limit (e.g., $+5^{\circ} \mathrm{C}$ above ambient) the test frequency shall be reduced to avoid effects from heating due to energy dissipation. Unless cyclic fatigue fracture tests are operated at resonance, e.g., in the $\mathrm{kHz}$ range [56], the cyclic frequency will remain below $100 \mathrm{~Hz}$. Compared with 
this, the time-scale on which the microscopic damage mechanisms are active is at least four to five orders of magnitude lower. This indicates that the microscopic cracks eventually may occur more or less stochastically during cyclic fatigue loading. On the other hand, AE signal sampling rates of at least $5-10 \mathrm{MHz}$ are thus required in order to sufficiently capture the broad-band frequency spectrum of the waves generated by the microscopic damage mechanisms [54].

Ex-situ X-ray computed tomography images of selected slices cut from CFRP epoxy laminates after quasi-static and cyclic fatigue mode I fracture, respectively, obtained at Empa (unpublished results) seem to hint at evidence for micro-cracks occurring in the matrix or as debonding between fibre and matrix located around, but not connected with the main delamination, i.e., direct proof of the formation of the so-called fracture process zone. Such cracks could not be observed with X-ray radiography in the delamination in the in-situ tests using a contrast agent, since there is no connectivity with the specimen surface and hence the contrast agent could not penetrate. In principle, the micro-cracks in the fracture process zone around the delamination will contribute to the increasing compliance with increasing number of fatigue cycles and thus simulate a larger effective delamination length, i.e., constitute another source for scatter in the delamination length measurements.

\section{Mixed mode loading conditions}

With a proper physics basis, one can then further explore aspects such as mode-interaction in loading. Currently, mixed mode fatigue is phenomenologically described with empirical relations between the individual opening modes, i.e. mode I and mode II. Such phenomenological description ignores however, that the process of fracture in both modes is completely different. Where in mode I, the process zone is confined to the direct vicinity of the crack tip, making the crack increment the dominant energy dissipating fracture mechanism; in mode II the fracture process zone is elongated over a large area ahead of the crack tip in which microscopic shear cracks are formed. These cracks contribute significantly to the strain energy dissipation in mode II, while crack growth consists of the coalescence of the micro-cracks. One could therefore argue that in mode II, the crack length alone is insufficient to describe the fatigue damage.

Hence, describing mixed mode fatigue loading in agreement with the physics should acknowledge the contribution of these different process zones and their characteristic fracture phenomena [46].

\section{Conclusions}

This paper discusses various aspects related to the physics of fatigue fracture testing of CFRP composites based on experimental observations in recently reported fatigue fracture testing. It illustrates that through quantification of strain energy dissipation in relation to applied work, one can identify the physical influence of various aspects related to loading or environment on the crack growth results.

Through this understanding of the physics, one can define procedures to obtain data that can be used for safe composite structural design. To use these data for design requires the elimination of 
extrinsic scatter, without affecting intrinsic scatter related to material morphology and process inhomogeneity.

Without quantification of the physical influence of all parameters on the fatigue fracture testing, the scatter in data will remain unacceptably large. It may even be difficult to completely and clearly distinguish between extrinsic and intrinsic scatter, despite their definition provided in this paper. Therefore the emphasis in future research should be put in developing physical understanding of the phenomena and mechanisms at the different length- and time scales, and finally provide knowledge on the proper averaging over these scales.

The authors believe that the physics of microscopic processes can only be fully understood if the macroscopic physics of fatigue loading is completely understood. Hence, instead of phenomenologically correlating observed crack growth rates against fracture mechanics parameters, one should quantify the fracture resistance in agreement with physics, i.e. dU/dA. Only in this way, one can potentially bridge the length- and time scales, relating macroscopic observations or measurements with measurements from e.g. acoustic emissions, $\mathrm{x}$-ray radiography, or X-ray computed tomography.

Additionally, the authors recommend further developing and evaluating approaches that quantify the contribution of fibre bridging unambiguously to use data obtained from unidirectional CFRP for structural design with multidirectional lay-ups and interfaces.

\section{Acknowledgments}

This paper focuses on selected questions and contributions that were discussed at a workshop on the "Physics of fatigue damage growth" held on the $10^{\text {th }}$ and $11^{\text {th }}$ of October 2016 at TU Delft with participants from several laboratories covering expertise on fatigue fracture testing of both metals and polymer composites: Dr. René Alderliesten, TUD/LR, Dr. Emiel Amsterdam, NLR, Prof. Rinze Benedictus, TUD/LR, Dr. Andreas Brunner, Empa, Prof. Masaki Hojo, Kyoto University, Prof. Neil James, Plymouth University, Dr. Frans v/d Meer, TUD / CITG, Dr. Silvain Michel, Empa, Prof. Wim van Paepegem, Gent University, Dr. John-Alan Pascoe TUD/LR, Prof. Mohan Ranganathan, Université François-Rabelais Tours, Prof. Marino Quaresimin, University of Padova, Dr. Calvin Rans, TUD/LR, Ir. Ruben Sevenois, Gent University, Dr. Gerben Sinnema, ESA / ESTEC, Dr. Sofia Teixeira de Freitas, TUD/LR, Dr. Enrico Troiani, Bologna University, Dr. Anastasios Vassilopoulos, EPFL, Dr. A.K. Vasudevan, US Navy/Office Naval Research. The discussion and authors 'views in this paper are not necessarily shared by all participants, but the contributions and the discussions among all participants are gratefully acknowledged.

\section{References}

1. Paris, P.C. (1963), The fracture mechanics approach to fatigue. in 10th Sagamore Army Materials Research Conference. Raquette Lake, NY: Syracuse University Press. 
2. Paris, P.C., M.P. Gomez, and W.E. Anderson (1961), A Rational Analytic theory of fatigue. The Trend in Engineering 13: 9-14.

3. Paris, P.C., G. Sih. (1965), Stress Analysis of Cracks. in Fracture Toughness Testing and its Applications, ASTM STP 381. Philadelphia, Pa.: American Society for Testing and Materials.

4. ASTM D6115-97 (2011), Standard test method for mode I fatigue delamination growth onset of unidirectional fiber-reinforced polymer matrix composites.

5. ASTM D5528-13 (2013), Standard Test Method for Mode I Interlaminar Fracture Toughness of Unidirectional Fiber-Reinforced Polymer Matrix Composites.

6. ISO 15024 (2001), Fibre-reinforced plastic composites -- Determination of mode I interlaminar fracture toughness, $G_{I C}$, for unidirectionally reinforced materials.

7. ASTM D7905-14 (2014), Standard Test Method for Determination of the Mode II Interlaminar Fracture Toughness of Unidirectional Fiber-Reinforced Polymer Matrix Composites.

8. ISO 15114 (2014), Fibre-reinforced plastic composites - Determination of the mode II fracture resistance for unidirectionally reinforced materials using the calibrated end-loaded split (C-ELS) test and an effective crack length approach.

9. ASTM D6671/D6671M - 13'1 D6671-06 (2006), Standard Test Method for Mixed Mode I-Mode II Interlaminar Fracture Toughness of Unidirectional Fiber Reinforced Polymer Matrix Composites.

10. Jones, R., A.J. Kinloch, W. Hu (2016), Cyclic-fatigue crack growth in composite and adhesivelybonded structures: The FAA slow crack growth approach to certification and the problem of similitude. Int. J. Fatigue 88: 10-18.

11. Rans, C.D., R.C. Alderliesten, R. Benedictus (2011), Misinterpreting the results: How similitude can improve our understanding of fatigue delamination growth, Compos. Sci. Technol. 71: 230238.

12. Alderliesten, R.C. (2016), How proper similitude can improve our understanding of crack closure and plasticity in fatigue, Int. J. Fatigue 82: 263-273.

13. Cano, A.J., A. Salazar, J. Rodriguez (2018), Evaluation of different crack driving forces for describing the fatigue crack growth behaviour of PET-G, Int. J. Fatigue 107: 27-32.

14. Brunner, A.J., S. Stelzer, G. Pinter, G.P. Terrasi (2016), Cyclic fatigue delamination of carbon fiber-reinforced polymer-matrix composites: Data analysis and design considerations. Int. J. Fatigue 83(2): 293-299.

15. Yao, L., R.C. Alderliesten, M. Zhao, R. Benedictus (2014)., Bridging effect on mode I fatigue delamination behavior in composite laminates. Composites Part A: Applied Science and Manufacturing 63: 103-109. doi:10.1016/j.compositesa.2014.04.007

16. Jones, R., R.K. Singh Rama, A.J. McMillan (2018), Crack growth: Does microstructure play a role?, Engineering Fracture Mechanics, 187: 190-210. doi:10.1016/j.engfracmech.2017.11.023 
17. ASTM E647-15 ${ }^{\mathrm{e} 1}$ (2015), Standard Test Method for Measurement of Fatigue Crack Growth Rates. American Society for Materials and Testing, ASTM International, West Conshohocken, USA: 1-49, doi: 10.1520/E0647-15E01

18. Jones, R., A.J. Kinloch, J.G... Michopoulos, A.J. Brunner, N. Phan (2017), Delamination growth in polymer-matrix fibre composites and the use of fracture mechanics data for material characterisation and life prediction. Composite Structures, 180: 316-333. doi: 10.1016/j.compstruct.2017.07.097

19.- Molent, L., C.Forrester (2017), The lead crack concept applied to defect growth in aircraft composite structures. Composite Structures ,166: 22-26. doi:

10.1016/j.compstruct.2016.12.076

20.- Composite Materials Handbook, Volume 3: Polymer Matrix Composite Materials Useage, Design and Analysis (2012), SAE International, ISBN 978-0-7680-7813-8, 1-954.

21. Grimes, G.C., E.G. Dusablon, R.L. Malone, J.P. Buban (1993) Tape Composite Materials Allowables Application in Airframe Design/Analysis. Composites Engineering, 3(7-8), 777-804.

22. Jones, R., S. Pitt, A.J. Brunner, D. Hui (2012), Application of the Hartman-Schijve equation to represent Mode $\{1\}$ and Mode $\{I\}\}$ fatigue delamination growth in composites, Compos. Struct. 94: 1343-1351. doi:10.1016/j.compstruct.2011.11.030.

17. Hojo, M., K. Tanaka, C.G. Gustafson, R. Hayashi, (1987), Effect of stress ratio on near-threshold propagation of delamination fatigue cracks in unidirectional CFRP, Compos. Sci. Technol. 29: 273-292.

24. Hojo, M., S. Ochiai, C.G. Gustafson, K. Tanaka (1994), Effect of matrix resin on delamination fatigue crack growth in CFRP laminates, Eng. Fract. Mech. 49(1): 35-47.

25. Atodaria, D.R., S.K. Putatunda, P.K. Mallick (1999), Delamination growth behaviour of a fabric reinforced laminated composite under mode I loading, J. Eng. Mater. Technol. 121(3): 381-385.

26. Khan, R. (2013), Delamination Growth in Composites under Fatigue Loading, Delft University of Technology. doi:10.4233/uuid:dbd8025c-c660-452b-8b0b-391c8ad3a89f

18. Khan, R., R.C. Alderliesten, L. Yao, R. Benedictus (2014), Crack closure and fibre bridging during delamination growth in carbon fibre/epoxy laminates under mode I fatigue loading, Composites: Part A 67: 201-211.

28. Khan, R., R.C. Alderliesten, S. Badshah, M.A. Khattak, M.S. Khan, R. Benedictus (2016), Experimental investigation of the microscopic damage development at mode I fatigue delamination tips in carbon/epoxy laminates, Jurnal Teknologi 78(11): 33-40.

29. Yao, L., R.C. Alderliesten, R. Benedictus (2015), Interpreting the stress ratio effect on delamination growth in composite laminates using the concept of fatigue fracture toughness, Composites: Part A 78: 135-142.

30. Stelzer, S., Brunner, A.J., Argüelles, A., Murphy, N., Pinter, g. (2012) Mode I delamination fatigue crack growth in unidirectional fiber reinforced composites: Development of a standardized test 
procedure. Composites Science and Technology 72: 1102-1107.

doi:10.1016/j.compscitech.2011.11.033

31. Stelzer, S., A.J. Brunner, A. Argüelles, N. Murphy, G.M. Cano, G. Pinter (2014), Mode I delamination fatigue crack growth in unidirectional fiber reinforced composites: Results from ESIS TC4 round-robins, Eng. Fract. Mech. 116: 92-107. https://doi.org/10.1016/j.engfracmech.2013.12.002

32. Murri, G.B. (2014) Effect of data reduction and fiber-bridging on Mode I delamination characterization of unidirectional composites. Journal of Composite Materials 48(19): 24132424. doi: $10.1177 / 0021998313498791$

33. Ray, B.C., S.T. hasan, D.W. Clegg (2007) Evaluation of Defects in FRP Composites by NDT Techniques. Journal of Reinfirced Plastics and Composites, 26(12): 1187-1192. doi: 10.1177/0731684407079348

34. Hsiao, K.-T. D. Heider (2017) Vacuum assisted resin transfer molding (VARTM) in polymer matrix composites, Chapter 10 in: Manufacturing Techniques for Polymer Matrix Composites (PMCs), (eds. S.G. Advani, K.-T. Hsiao), Woodhead Publishing, ISBN: 9780857090676, 310-347.

35. D.J. Nicholls and J.P. Gallagher (1983) Determination of GIC in angle ply composites using a cantilever beam test method, J. Reinf. Plast. Comp. 2/1: 2-17. doi:10.1177/073168448300200101

36. Choi, N.S., Kinloch, A.J., Williams, J.G. (1999) Delamination Fracture of Multidirectional CarbonFiber/Epoxy Composites under Mode I, Mode II and Mixed-Mode I/II Loading. Journal of Composite Materials 33: 73-100. doi: 10.1177/002199839903300105

37. Gong, X.J., Hurez, A., Verchery, G. (2010) On the determination of delamination toughness by using multidirectional DCB specimens. Polymer Testing, 29: 658-666. doi:10.1016/j.polymertesting.2010.04.007

38. Sebaey, T.A., Blanco, N., Costa, J., Lopes, C.S. (2012) Characterization of crack propagation in mode I delamination of multidirectional CFRP laminates. Composites Science and Technology, 72: 1251-1256. doi: 10.1016/j.compscitech.2012.04.011

39. Hojo, M., T. Aoki (2015), Characterization of fatigue R-curves based on Gmax-constant delamination tests in CF/PEEK laminates, Proceedings 20th International Conference on Composite Materials, Copenhagen, 19-24th July 2015, 1-8.

40. Griffith, A.A. (1921), The phenomena of rupture and flow in solids. Philos Trans R Soc London Ser A, Contain Papers Math Phys Character 221:163-98.

41. Pascoe, J.A. (2016), Characterisation of Fatigue Crack Growth in Adhesive Bonds, Delft University of Technology. doi:10.4233/uuid:ebbf552a-ce98-4ab6-b9cc-0b939e12ba8b.

42. Amaral, L., L. Yao, R.C. Alderliesten, R. Benedictus (2015), The relation between the strain energy release in fatigue and quasi-static crack growth, Eng. Fract. Mech. 145: 86-97. 
43. Yao, L., R.C. Alderliesten, M. Zhao, R. Benedictus (2014)., Discussion on the use of the strain energy release rate for fatigue delamination characterization. Composites Part A: Applied Science and Manufacturing 66: 65-76.

44. Pascoe, J.A., R.C. Alderliesten, R. Benedictus (2017), On the physical interpretation of the R-ratio effect and the LEFM parameters used for fatigue crack growth in adhesive bonds, Int. J. Fatigue 97: 162-176. doi:10.1016/j.ijfatigue.2016.12.033.

45. Hartman A, Schijve J. (1970), The effects of environment and load frequency on the crack propagation law for macro fatigue crack growth in aluminum alloys. Eng. Fract. Mech. 1(4): 615-631.

46. Amaral, L., R.C. Alderliesten, R. Benedictus (2017), Understanding mixed-mode cyclic fatigue delamination growth in unidirectional composites: An experimental approach, Eng. Fract. Mech. 180: 161-178.

47. Amaral, L., Zarouchas, D., R.C. Alderliesten, R. Benedictus (2017), Energy dissipation in mode II fatigue crack growth, Eng. Fract. Mech. 173: 41-54.

48. Brunner A.J. (2016), Correlation between Acoustic Emission signals and delaminations in carbon fiber reinfored polymer-matrix composites: A new look at mode I fracture test data, Journal of Acoustic Emission, 33, S41-S46, available on: http://www.aewg.org/jaeonline.htm.

49. Garcea, S.C., M.N. Mavrogordato, A.E. Scott, I. Sinclair, S.M. Spearing (2014), Fatigue micromechanism characterisation in carbon fibre reinforced polymers using synchrotron radiation computed tomography, Compos. Sci. Technol. 99: 23-30

50. Garcea, S.C., I. Sinclair, S.M. Spearing (2015), In situ synchrotron tomographic evaluation of the effect of toughening strategies on fatigue micromechanisms in carbon fibre reinforced polymers, Compos. Sci. Technol. 109: 32-39.

51. Zarouchas, D.S., R.C. Alderliesten, J.A. Pascoe (2016): Dataset: Acoustic Emission during Crack Growth in FM94 Epoxy. https://doi.org/10.4121/uuid:8cb928b4-4dc9-4420-8adc-f1273c9fd7c5

52. Sause, M.G.R. (2016) In Situ Monitoring of Fiber-Reinforced Composites Theory, Basic Concepts, Methods, and Applications, Springer Series in Materials Science, 1-633, doi: 10.1007/978-3-31930954-5

53. ISO 14125 (1998), Fibre-reinforced plastic composites -- Determination of flexural properties.

54. Sause, M.G.R., S. Horn (2010) Simulation of Acoustic Emission in Planar Carbon Fiber Reinforced Plastic Specimens, Journal of Nondestructive Evaluation 29: 123-142, doi: 10.1007/s10921-0100071-7

55. https://www.comsol.com/structural-mechanics-module

196. Maillet, I., L. Michel, F. Souric, Y. Gouri (2015) Mode II fatigue delamination growth characterization of a carbon/epoxy laminate at high frequency under vibration loading, Engineering Fracture Mechanics 149: 298-312. 10.1016/j.engfracmech.2015.08.030 